in particular in relation to people with more than two of these experiences and for health outcomes other than infections. This situation is exacerbated by the under-ascertainment of these populations in routine information sources on population health needs, such as surveys and censuses. In many countries, administrative data are available which could help address these knowledge gaps. We describe the creation and characteristics of a novel virtual cohort using cross-sectoral linkage of administrative datasets, in order to inform policy and practice responses to these co-occurring issues.

Methods Individual-level data from local authority homelessness services (HL), opioid substitution therapy dispensing (OST), and a psychosis case register (PSY) in Glasgow, Scotland between 2011-15 were confidentially linked to National Health Service records, using a mix of probabilistic and deterministic linkage. A de-identified dataset was made available to researchers through a secure analysis platform. Demographic characteristics associated with different exposure combinations were analysed using descriptive statistics.

Results Linkage created a cohort of 24,767 unique individuals with any one of the experiences of interest between 2011-15. Preliminary results suggest that $89.2 \%$ of the cohort had one experience; $10.6 \%$ two; and $0.2 \%$ all three. The most common combination was HL \& OST ( $\mathrm{O}=2,150 ; 8.7 \%)$, with other combinations much less frequent (HL \& PSY, $n=279$, $1.1 \%$; OST \& PSY, $\mathrm{n}=188,0.8 \%$; HL \& OST \& PSY, $\mathrm{n}=51$, $0.2 \%)$. The odds of male gender increased with number of exposures (2 exposures, OR 2.1, 95\% CI 1.9-2.2; 3 exposures, OR 4.1, 95\% CI 2.3-7.2), but there was little difference in age. Work is ongoing to incorporate into the cohort additional datasets on criminal justice involvement.

Discussion Administrative data linkage is a feasible approach to understanding the health of people affected by multiple exclusionary processes, addressing problems of recruitment and retention affecting traditional cohort studies in this field. As well as improving the validity of descriptive epidemiology for these populations, this study offers a foundation for evaluating future policy or service interventions. In order for the benefits of administrative data research to be realised, robust and timely governance and linkage processes are required.

\section{OP21 INEQUALITIES IN THE PREVALENCE AND DEVELOPMENT OF MULTIMORBIDITY ACROSS ADULTHOOD: FINDINGS FROM THE 1946 NATIONAL SURVEY OF HEALTH \& DEVELOPMENT}

\footnotetext{
${ }^{1,2}$ AR Khanolkar*, ${ }^{1} \mathrm{~N}$ Chaturvedi, ${ }^{1} \mathrm{D}$ Davis, ${ }^{1} \mathrm{~A}$ Hughes, ${ }^{1} \mathrm{M}$ Richards, ${ }^{3} \mathrm{~V}$ Kuan Po Ai, ${ }^{4} \mathrm{D}$ Bann, ${ }^{1,4} \mathrm{P}$ Patalay. ${ }^{1} \mathrm{MRC}$ Unit for Lifelong Health and Ageing at UCL, University College London, London, UK; ${ }^{2}$ Institute for Environmental Medicine, Karolinska Institutet, Stockholm, Sweden; ${ }^{3}$ Institute of Health Informatics, University College London, London, UK; ${ }^{4}$ Centre for Longitudinal Studies, University College London, London, UK
}

\subsection{6/jech-2020-SSMabstracts.21}

Background With increasing life expectancy and aging populations, the prevalence of multimorbidity (two or more conditions in a person) is rising. Multimorbidity is progressively more common in older age, socioeconomically disadvantaged groups and associated with increased mortality and morbidity. Most studies on multimorbidity to date are cross-sectional in design or with limited follow-up.

We studied the development of multimorbidity across adulthood and early old age in a nationally representative birth cohort study.
Methods This study analysed data from the 1946 National Survey of Health and Development (NSHD). The analysis sample included participants who attended the age 36 assessment in 1982 and any one of the follow-up assessments (ages 43, 53, $63 \& 69 ; \mathrm{N}=3,723,51 \%$ males). Information on 18 common conditions was based on a combination of selfreport, prescribed medications and health records.

Conditions included diabetes, dyslipidaemia, hypertension, obesity, coronary heart disease, stroke, cancer, anaemia, respiratory-, kidney-, gastro intestinal-, skin-disorders, arthritis, Parkinson's disease, epilepsy, depression \& psychosis.

For all participants, we calculated a multimorbidity score at each age indicating the number of conditions accumulated over time and was the outcome of interest. Linear-spline mixed-effects modelling was used to study the population-average accumulation of conditions over time in different periods. We also assessed sex and socioeconomic differences in longitudinal trajectories of multimorbidity across the five ages (18,615 data points, mean: 5 data points/participant).

Childhood social class and adulthood educational level were used as socioeconomic indicators. Missing data was addressed using multiple imputation.

Results Proportion of participants with no conditions decreased progressively from $52 \%$ at age 36 to $7 \%$ at age 69 . Multimorbidity (the number of conditions) increased progressively across all 4 periods $(0.55,95 \%$ CI $[0.5,0.6]$ for $1982-$ $89,0.63[0.58,0.7]$ for $1989-99,0.70$ [0.63, 0.78] for 1999-09 and 1.15 [1.04, 1.25] for 2009-15). Disadvantaged social class in childhood was associated with marginally increased multimorbidity in adulthood $(0.08$ [0.01, 0.15] for skilled/unskilled and 0.07 [-0.01, 0.15] for manual groups compared to professional/intermediate group). Higher educational attainment was associated with decreased risk for multimorbidity $(-0.09[-0.2,0.01]$ for university degree and -0.13 $[-0.2,-0.06]$ for General Certificate of Education (GCE) compared to those without education). Estimates for childhood social class were attenuated and no longer significant when adjusted for educational level. Women had marginally higher risk for multimorbidity compared to men $(0.15$ [0.09-0.2]).

Conclusion Multimorbidity increases progressively with age, with the socioeconomically more disadvantaged having greater multimorbidity.

\section{OP22 UNDERSTANDING PATHWAYS TO HEALTH INEQUALITIES IN CYSTIC FIBROSIS - A CAUSAL MEDIATION ANALYSIS USING UK REGISTRY DATA}

${ }^{1}$ DK Schlüter*, ${ }^{2} R$ Keogh, ${ }^{3} S$ Agbla, ${ }^{4} R$ Daniel, ${ }^{1} D$ Taylor-Robinson. ${ }^{1}$ Public Health and Policy, University of Liverpool, Liverpool, UK; ${ }^{2}$ Medical Statistics, London School of Hygiene and Tropical Medicine, London, UK; ${ }^{3}$ Biostatistics, University of Liverpool, Liverpool, UK; ${ }^{4}$ School of Medicine, Cardiff University, Cardiff, UK

\subsection{6/jech-2020-SSMabstracts.22}

Background Cystic fibrosis (CF) is an inherited, progressive condition affecting over 10000 individuals in the UK. Symptoms of CF include poor growth, lung infection, poor lung function and reduced survival. Outcomes are worse for people growing up in disadvantaged circumstances, but it is not clear to what extent inequalities in early growth can explain inequalities in later lung function and survival. Our aim was to assess how the association between socioeconomic circumstances (SECs) and subsequent lung function measured around age 6 is mediated by weight trajectory in early childhood. 
Methods We did a causal mediation analysis using data from the UK CF registry, which captures 99\% of all people with CF in the UK and records clinical information including weight and infection status at annual review visits. The exposure of interest was SEC in the first year of life measured by the index of multiple deprivation; the outcome was first lung function measure between ages 6 and 9. We were interested in mediation by weight trajectory during the first six years of life. Potential confounders were sex, year of birth, genotype and infection.

All children born between 2000 and 2010 and diagnosed by newborn screening were included in the analysis if they had at least one lung function measure between ages 6 and 9, at least one weight and infection measure between birth and age 6, and complete data on SECs and baseline confounders. We imputed missing data using multiple imputation by chained equations.

We used the parametric mediational g-formula to estimate the total effect of SECs on lung function, and the indirect effect mediated by weight trajectories in the first six years of life, accounting for potential time-varying confounding by infection. Confidence intervals were estimated using non-parametric bootstrap.

Results Using data from 853 children, we found a total effect of deprivation on lung function, measured by percent of predicted FEV1, of 4.53 percentage points (95\% CI 3.44 to 5.77). Our results showed that if we could improve the weight of the most disadvantaged children to have the same distribution as that of the least disadvantaged children, their lung function would improve on average by 0.74 percentage points (95\% CI $0.36-1.1)$.

Conclusion Only 16\% (95\% CI 8\%-25\%) of the inequalities in early lung function for people with CF were explained by weight trajectories in the first 6 years of life, suggesting that other important pathways to inequalities need exploration.

\section{Wednesday 9 September}

\section{Life Course: Childhood}

\section{OP23 THE LESSER-KNOWN BREASTFEEDING PROBLEM: PREVALENCE AND DETERMINANTS OF PRELACTEAL FEEDING PRACTICE IN INDONESIA}

LD Rahmartani*, C Carson, MA Quigley. Nuffield Department of Population Health, University of Oxford, Oxford, UK

10.1136/jech-2020-SSMabstracts.23

Background Prelacteal feeding (PLF) is anything other than breastmilk given to neonates before breastfeeding is established. There are numerous types of PLF and they are given for various reasons. Except when medically indicated, PLF is considered as one of the many potential barriers to optimal breastfeeding. However, PLF is practiced widely across the world. Meanwhile, it is still understudied and epidemiological research on the different types of PLF is limited in many settings, including in Indonesia. This study looks at the prevalence and determinants PLF in Indonesia, focusing on overall PLF and three common types (formula, other milk, and honey).

Methods A cross-sectional, secondary data analysis of the 2017 Indonesia Demographic and Health Survey was undertaken. The study population was 6168 ever-breastfeeding mothers whose last child was $\leq 23$ month-old. Because PLF was a common outcome, modified Poisson regression was used to estimate the adjusted prevalence ratio (PR) for potential determinants and PLF.

Results By 2017, nearly half (45\%) of mothers in Indonesia gave PLF. The most common feeds were infant formula (25\%), any other milk (14\%), plain water $(5 \%)$, and honey (3\%). Factors associated with higher prevalence of overall PLF were upper-middle (Q3-Q4) wealth quintiles (PR 1.17, 95\% Confidence Interval (CI) 1.03-1.32 for Q3 and PR 1.18, 95\% CI 1.04-1.33 for Q4), rural residence (PR 1.17, 95\%CI 1.071.27), baby perceived to be small at birth (PR 1.26, 95\%CI 1.14-1.38), and caesarean deliveries at either public (PR 1.34, 95\%CI 1.19-1.51) or private facilities (PR 1.17, 95\%CI 1.031.33). Conversely, mothers who gave birth to the second/subsequent child (PR 0.81, 95\%CI 0.75-0.87) and mothers who possessed an antenatal card (PR 0.86, 95\%CI 0.77-0.96) were less likely to give PLF. When analysed separately, formula displayed relatively similar risk factors to those of overall PLF, yet several associations varied among these three types of PLF. For instance, higher wealth quintiles and rural residence were risk factors for formula but not for other milk and honey. Furthermore, honey was more prevalent in home births than in deliveries at health facilities (PR 6.05, 95\% CI 4.02-9.10), but formula and other milk were more frequent among caesarean deliveries. First-time birth was the only factor that showed a consistent association with overall PLF, formula, other milk, and honey.

Conclusion PLF is common in Indonesia but the prevalence and determinants vary by PLF type. Identifying high-risk groups, particularly by PLF type, is useful to plan more targeted interventions to improve breastfeeding practices.

\section{OP24 THE MEDIATING PATHWAYS OF THE ASSOCIATION BETWEEN ADVERSE CHILDHOOD EXPERIENCES AND COGNITIVE HEALTH IN LATER LIFE}

${ }^{1} \mathrm{D}$ Cadar, ${ }^{2}$ LK Kobayashi, ${ }^{1} \mathrm{AP}$ Steptoe, ${ }^{3} \mathrm{P}$ Demakakos*. ${ }^{1}$ Department of Behavioural Science and Health, University College London, London, UK; ${ }^{2}$ Department of Epidemiology, University of Michigan School of Public Health, Ann Arbor, Michigan, USA; ${ }^{3}$ Department of Epidemiology and Public Health, University College London, London, UK

\subsection{6/jech-2020-SSMabstracts.24}

Background Previous research has often shown that morbidity and disability are hinged to negative events and exposures that can accumulate over the life course, but less clear is their impact on late-life cognitive health. We assessed the biopsychosocial mechanisms influencing the associations between adverse childhood experiences and cognitive impairment at advanced ages.

Methods Data were from 3,130 dementia-free adults aged 50 + from the English Longitudinal Study of Ageing (ELSA) with data available from wave $3(2006 / 07)$ to wave $8(2016 / 17)$. ELSA provides a wide variety of psychosocial data collected via face-to-face computer-assisted personal interviews (CAPI) and self-completion questionnaires. All participants provided informed consent prior to their participation in the study. We used structural equation modelling to estimate direct and indirect associations between adverse childhood experiences (ACEs) and cognitive impairment (measured with $1.5 \mathrm{SD}$ below the mean on the modified Telephone Cognitive Screening Interview scale range 0 to 35 ) via markers of SES (education and wealth), inflammation (serum fibrinogen and C- 